\title{
EFFECTS OF LOW TEMPERATURE AND ELECTRICAL (SQUARE WAVE) STIMULATION ON SPONTANEOUS CONTRACTIONS IN ISOLATED GUINEA PIG ATRIA AND INFLUENCE OF THIAMINE TETRAHYDROFURFURYL DISULFIDE (TTFD) ON THESE EFFECTS
}

\author{
Hideo SHINOZAKI ${ }^{1}$ and Jiro Sugimoto ${ }^{2}$ \\ ${ }^{1}$ Biological Research Laboratories, Central Research Division, \\ Takeda Chemical Industries, Ltd. \\ ${ }^{2}$ Department of Pharmacology, Kansai Medical School, \\ Moriguchi-City 570, Japan
}

(Received September 10, 1975)

\begin{abstract}
Summary 1. The spontaneous contractions of isolated guinea pig atria were arrested by a temperature change in the medium from 30 to $20^{\circ} \mathrm{C}$ within $20 \mathrm{~min}$.

2. When thiamine tetrahydrofurfuryl disulfide (TTFD) at concentration of $10^{-5} \mathrm{~g} / \mathrm{ml}$ was added in the medium, the arrest was not seen for more than $30 \mathrm{~min}$.

3. Arrhythmic contractions induced by the electrical square wave stimulation at threshold intensity were prevented by TTFD at $10^{-4} \mathrm{~g} / \mathrm{ml}$ which was added to the medium.

4. These effects of TTFD at respective concentrations were seen even 30 min after the drug was removed from the medium, when the atria had been pre-incubated with the drug for one hour before the removal.

5. From these results, it was assumed that TTFD might show these effects against the extrinsic physical invasions through the stabilization of the tissue membrane.
\end{abstract}

In 1972, NAGATA et al. (1) reported that the spontaneous contractions of isolated guinea pig atria were arrested after a proper period of preparation, the potassium ion content was decreased and the sodium ion content increased when the preparation was contracted in a potassium-free Locke's solution, and that the time required to arrest the atrial contractions was extended and the change of the potassium ion content was prevented when thiamine tetrahydrofurfuryl disulfide (TTFD) was added to the potassium-free Locke's solution. In the same experiment, it was also found that dimethialium propyldisulfide could extend the time required to arrest the atrial contraction by the solution while decrease in potassium ion content was scarcely prevented by this drug.

\footnotetext{
1 篠崎英男, ${ }^{2}$ 杉本治良
} 
Subsequently, SUGImoto et al. (2) pointed out that these effects of TTFD remained even after the drug was removed from the medium by the replacement of the medium when the preparation had been pre-incubated with TTFD for one hour and that a large amount of thiamine was found in the prepared tissue after the incubation with TTFD and the content of transformed thiamine was scarcely reduced by the replacement of the medium with TTFD-free Locke's solution.

In the same year, SUGImoтo et al. (3) presented the other effects of TTFD showing that the time required to arrest the atrial contraction by a toxic dose of strophanthin-G was extended and the decrease in potassium ion content induced by the drug was prevented when TTFD was added to the preparation together with strophanthin-G. It was also found that the effects of TTFD remained even after the drug had been removed from the medium.

As thiamine itself could scarcely shown these effects, it has been assumed that TTFD might have some kind of pharmacological action different from thiamine and that the drug probably have some kind of the preventive effect against extrinsic stimulation or invasion given on the isolated atria. Then, the authors investigated the effects of TTFD on arrhythmic contraction induced by electrical stimulation in isolated guinea pig atria and on the arrest of the contraction induced by changing the medium temperature.

\section{MATERIALS AND METHODS}

Adult male guinea pigs weighing 350 to $450 \mathrm{~g}$ were used. After sacrificing by decapitation, the chest was opened, the heart removed, and the atria separated from the ventricles. The isolated atria were suspended in a Magnus' apparatus in a Locke's solution saturated with oxygen at 30 to $31^{\circ} \mathrm{C}$. The spontaneous contractions were recorded on a smoked drum using a spring lever or on an electrical recorder using of a strain-gauge (UL-2, Shinkotsushin).

Two kinds of stimulation were given to the preparation: one was low or high temperature stimulation and the other was electrical stimulation. The recording of the preparation response to the former stimulation was made using a smoked drum and that to the latter was made using an electrical recorder (AS-3A, Shinkotsushin). The electrical stimulation was given to the preparation by use of electronic stimulator (MSE-3R, Nihonkohden) through field platinum electrodes for a duration of $5 \mathrm{msec}$, at 120 cycles per minute and with three kinds of intensities, i.e., the threshold voltage to induce the arrhythmic contraction and those at 0.5 volts lower and higher than the threshold.

The Locke's solution used consisted of $\mathrm{NaCl} 9.0 \mathrm{~g}, \mathrm{KCl} 0.42 \mathrm{~g}, \mathrm{CaCl}_{2} 0.24 \mathrm{~g}$, $\mathrm{NaHCO}_{3} 0.7 \mathrm{~g}$, and glucose $1.0 \mathrm{~g}$ per liter of distilled water. The drug was added to the medium containing the atrial preparation, and the drug concentration in the medium was expressed by terms of $\mathrm{g} / \mathrm{ml}$. Thiamine tetrahydrofurfuryl disulfide $\cdot \mathrm{HCl}$ (TTFD) used in the present experiment was supplied by Takeda Chemical Industries. 


\section{RESULTS}

1. The effect of low temperature on spontaneous contractions of guinea pig atria and the influence of TTFD on it

When the temperature in the medium was suddenly lowered from 30 to $20^{\circ} \mathrm{C}$, the rate of contractions were reduced and contractile tension increased. The degrees of changes in rate and tension augmented with the lapse of time for 5 to $15 \mathrm{~min}$, and the contractions, thereafter, suddenly became arrhythmic when the rate reduced to less than $50 \%$ per minute. In every case, the arrhythmic contractions continued for 3 to $5 \mathrm{~min}$, and then arrested completely within $20 \mathrm{~min}$ after the temperature change. When the temperature was lowered to $18^{\circ} \mathrm{C}$ in place of $20^{\circ} \mathrm{C}$, contractions were often arrested within $3 \mathrm{~min}$.

When TTFD at $10^{-4}$ or $10^{-5}$ was added to the medium one hour before the temperature change, rhythmic contractions continued for more than $30 \mathrm{~min}$ : for 5 to $15 \mathrm{~min}$ after the temperature change, the changes of rate and tension of contractions were similar to those of the control cases, but, thereafter, neither arrhythmic contraction nor contraction arrest was seen for $60 \mathrm{~min}$ (Fig. 1). When TTFD $10^{-6}$ was used in place of $10^{-4}$ or $10^{-5}$, the effect of TTFD was limited. And, when the temperature was changed from 30 to $18^{\circ} \mathrm{C}$, TTFD was not effective at any concentration.

These effects of TTFD $10^{-4}$ or $10^{-5}$ was also seen even at 30 to 60 min after the drug was removed from the medium, when the preparation had been previously incubated with the drug for one hour before the removal (Fig. 2).

2. The effect of high temperature on spontaneous contractions of guinea pig atria and the influence of TTFD on it

When the medium temperature was increased from 30 to $35^{\circ} \mathrm{C}$, the rate of contractions was immediately increased to 130 to $160 \%$ and the tension was decreased to 40 to $50 \%$ of those of the controls at $30 \mathrm{~min}$ after the temperature change. When the temperature was changed from 30 to $40^{\circ} \mathrm{C}$, the rate was increased to 160 to $200 \%$ and the tension was decreased to less than $10 \%$ of those of the controls already at $5 \mathrm{~min}$ after the temperature change, though the contraction arrest was not observed for $30 \mathrm{~min}$ in every case.

When TTFD $10^{-4}$ or $10^{-5}$ was added to the medium before the temperature change from 30 to $35^{\circ} \mathrm{C}$, the rate of contractions was increased to 150 to $200 \%$ and the tension was decreased to less than $10 \%$ of those of the controls at 5 min after the temperature change, so that the drug might augment the influence of temperature change on the atrial contractions. When TTFD at same concentrations were added before the temperature change from 30 to $40^{\circ} \mathrm{C}$, the influence of the temperature change on the rate and tension of contractions was almost same as that which was seen when the drug was not added. 
3. The influence of TTFD on the arrhythmic contraction induced by electrical stimulation in spontaneous contractions of guinea pig atria

The spontaneously contracting atria were stimulated by the electrical stimulation at 120 cycles per minute, at a duration of $5 \mathrm{msec}$, and at threshold and threshold \pm 0.5 volts intensities to induce arrhythmic contraction.

When TTFD $10^{-4}$ was added one hour before the electrical stimulation, the intensities of the stimulation required to induce the arrhythmic contraction in the preparation was increased by 0.5 volts (Fig. 3). This effect of TTFD was observed in every case when the drug was applied at $10^{-4}$, while the drug effect was evident only in 2 or 3 cases out of 10 cases when the drug was added at $10^{-5}$.

This effect of TTFD was seen even at 30 to $60 \mathrm{~min}$ after the drug was removed from the medium, when the preparation had been pre-incubated with the drug for one hour before drug removal.

\section{DISCUSSION}

The authors (1-3) have already reported two kinds of TTFD effects: one a preventive effect on isolated guinea pig atria from the influence of potassium-free Locke's solution and the other that from the influence of strophanthin-G. However, these influences were chemical invasions. In the present experiment, the investigations were performed to determine whether TTFD could prevent the atria from physical invasion or not. As the physical invasion, temperature change in the medium and electrical stimulation were used.

The arrest of spontaneous contractions of isolated atria induced by lowering the temperature from 30 to $20^{\circ} \mathrm{C}$ was prevented by the presence of TTFD in the medium. And, the threshold intensity of electrical stimulation to induce arrhythmic contraction on the atrial spontaneous rhythmic contractions was increased when TTFD was added before the stimulation. These findings may sug-

Fig. 1. Spontaneous contractions of isolate guinea pig atria: when the temperature of the medium was lowered from 30 to $20^{\circ} \mathrm{C}$, the contractions were arrested within 20 min after the temperature change, but, when TTFD was added at $10^{-5} \mathrm{~g} / \mathrm{ml}$, the effect of low temperature on the contractions was moderated and contraction was not arrested for more than $30 \mathrm{~min}$. Numerals above the record give frequencies of contractions per minute. (W) shows the exchange of bath fluid for Locke's solution.

Fig. 2. Spontaneous contractions of isolated guinea pig atria: the effect of TTFD shown in Fig. 1 was seen even after the added TTFD $10^{-5} \mathrm{~g} / \mathrm{ml}$ had been washed out from the medium, when the preparation had been pre-incubated with TTFD for one hour. Numerals above the record give frequencies of contractions per minute. (W) shows the exchange of bath fluid for Locke's solution.

Fig. 3. Spontaneous contractions of isolated guinea pig atria: when the electrical stimulation was given at $5 \mathrm{msec}$, at $120 \mathrm{cycles} / \mathrm{min}$, and at intensities of $2,2.5$, and 3 volts, the threshold intensity to induce arrhythmic contractions was increased by the pre-added TTFD $10^{-4} \mathrm{~g} / \mathrm{ml}$. Numerals above the record give frequencies of contractions per minute. (W) shows the exchange of bath fluid for Locke's solution. 


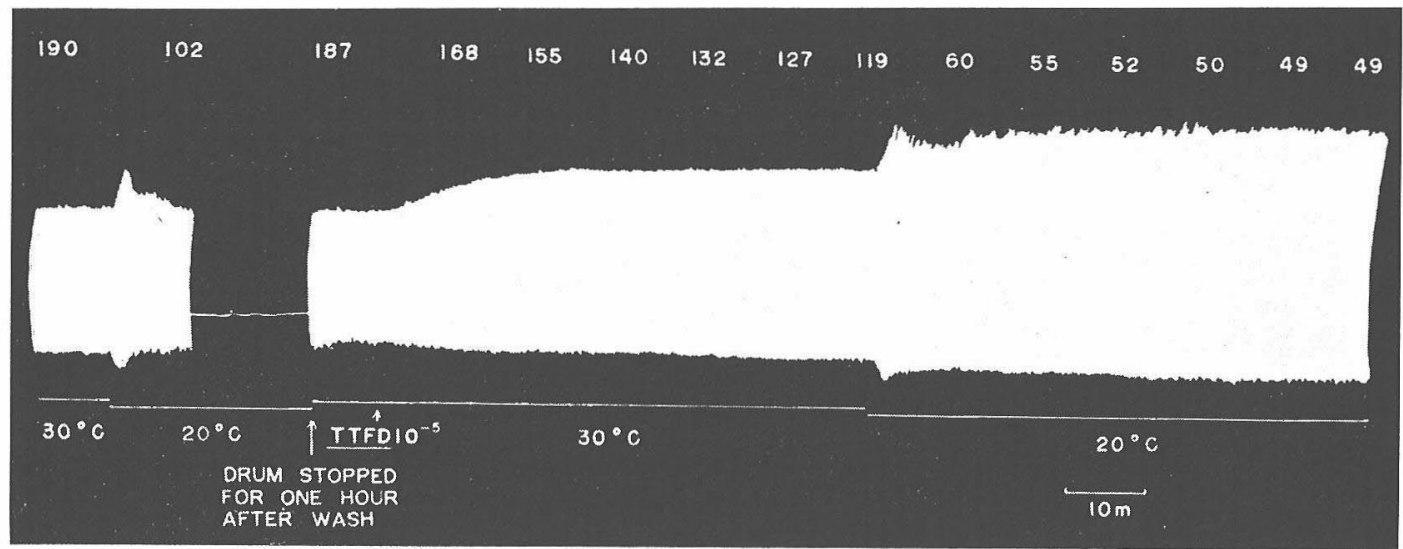

Fig. 1.

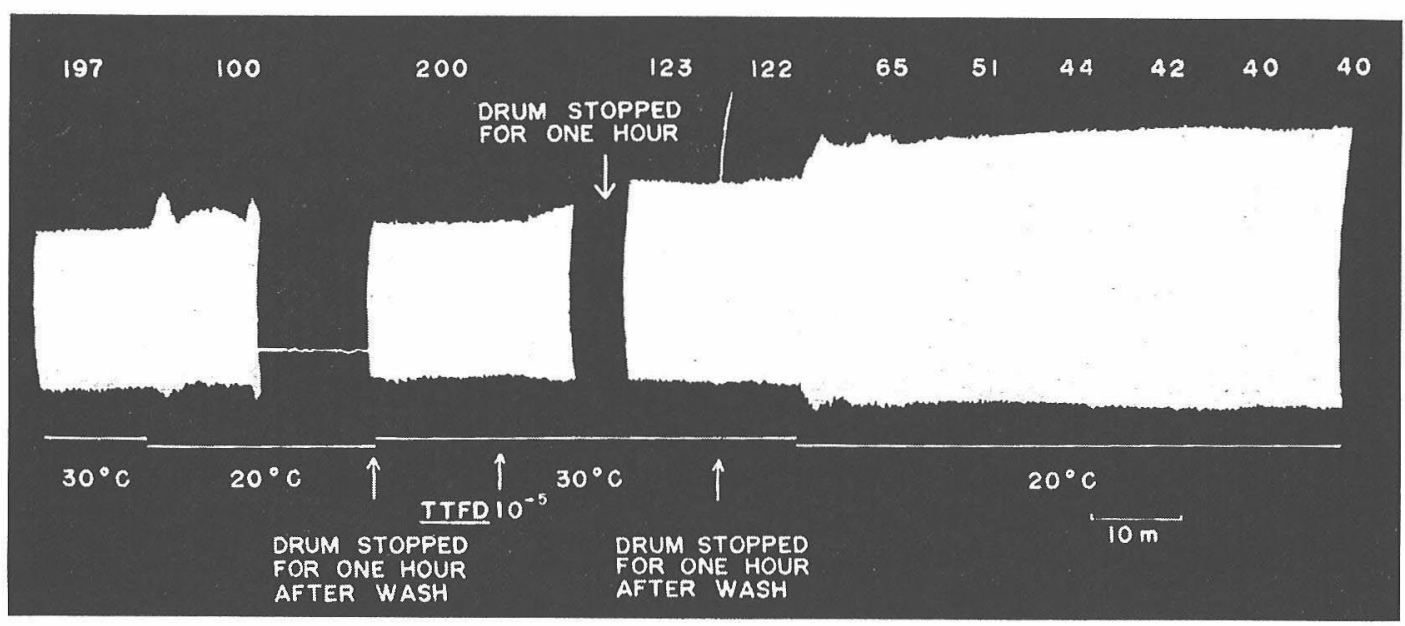

Fig. 2.
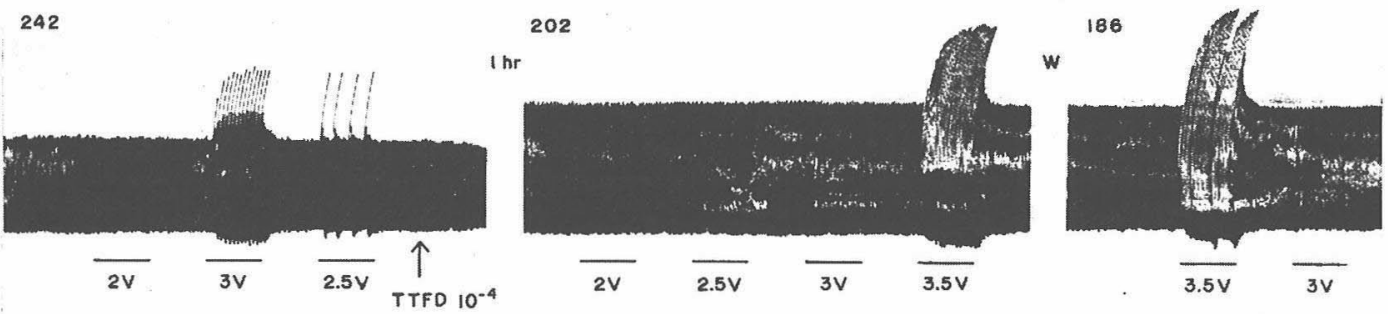

Fig. 3. 
gest that the drug might be able to prevent the atria not only from chemical invasions but also from physical invasions.

The effect of TTFD to prevent arrhythmic contraction induced by electrical stimulation on the isolated atrial contraction may suggest also the probable antiarrhythmic action in a cardiac function, because of its effect to prevent ectopic beats, and would support clinical experiences on anti-arrhythmic effect of TTFD reported by Miyake (4) and Kimura and Furukawa (5). However, the antiarrhythmic action of TTFD would be very weak, as the drug effects shown in the present experiment were not seen in low concentration but only in high concentration such as $10^{-5}$ or $10^{-4} \mathrm{~g} / \mathrm{ml}$.

It was interesting that the effects of TTFD to prevent the atria from the temperature change in the medium and the electrical stimulation were seen even after the drug was removed from the medium, when the preparation had been preincubated with the drug. Similar residual effect of TTFD was observed in our previous experiments, also, in which the effects of TTFD against influences of potassium-free medium and strophanthin- $G$ in a toxic dose on atria were investigated $(1,3)$. And, Sugimoto et al. (2) reported that a large amount of thiamine was found in atrial tissue after the preparation had been incubated with TTFD and that the biotransformed thiamine remained in the tissue even after the preparation was incubated with TTFD-free Locke's solution. However, it is not yet definitely decided whether these residual effects of TTFD are caused by the large amount of biotransformed thiamine or by some residual change in the tissue membrane induced by the added TTFD in the process of the biotransformation. In any case, it can be probably assumed that TTFD possesses an action which moderates the change of isolated tissue responses seen when either chemical or physical invasion was given from the outside, and that the drug effect might be long-lasting.

If TTFD is expected to be an anti-arrhythmic drug, its effects on the refractory period, the maximal driving frequency, and the other cardiac muscle functions have to be evaluated. These will be reported on in a succeeding paper.

The authors wish to thank Professor M. Morita for advice and constructive criticism.

\section{REFERENCES}

1) Nagata, M., Sugimoto, J., and Fugono, R., Jap. Circ. J., 36, 935 (1972).

2) Sugimoto, J., Nagata, M., and Fugono, R., Jap. Circ. J., 37, 135 (1973).

3) Sugimoto, J., Nagata, M., and Nishikubo, Y., Jap. Circ. J., 37, 491 (1973).

4) MiYaKe, Y., Shinryo (in Japanese), 17, 7 (1964).

5) Kimura, N. and Furukawa, I., Chiryo (in Japanese), 48, 20 (1966). 